PART 2

R CORONAE BOREALIS STARS 


\title{
THE R CORONAE BOREALIS TYPE VARIABLES
}

\author{
M. W. FEAST \\ South African Astronomical Observatory
}

(Read by T. Lloyd Evans)

\begin{abstract}
A general review of the RCB stars is given. The available observations (spectra, photometry (including infrared) and polarization) seem most easily explained by a model in which a cloud of dust is ejected towards the observer at times of deep minima. The large radius changes in the pulsation cycle of RY Sgr suggest a high luminosity. This is also indicated by the RCB stars in the LMC. Theoretical work on RCB stars is briefly reviewed. The possible relation of the variables to the planetary nebulae and the novae is mentioned.
\end{abstract}

\section{Introduction}

The RCB stars are a very small group $(\sim 30)$ of objects. As will be discussed, the evidence indicates that they are low-mass objects passing rapidly through a late stage of stellar evolution which is characterized by abnormal abundances, high luminosities and the ability to form and dissipate circumstellar dust. In addition some at least of these variables are also pulsating stars and this enables comparisons to be made with the predicted pulsational characteristics of hydrogen poor, helium and carbon rich, objects.

\section{Definition, Light Variations, Distribution}

The RCB stars spend much of their time near maximum light with occasional drops in brightness which range from small dips to major declines of 7 mag. or even more lasting several years. All well-authenticated RCB stars have spectra which indicate that they are hydrogen poor, carbon rich objects and it seems very advisable to add this as a defining characteristic of the group. This enables one to discard, on the basis of their spectra, a number of stars which from time to time have been placed incorrectly in this class. On more detailed examination such stars have generally turned out to belong to some other well known class of variables (e.g. eclipsing, long period, cataclysmic).

There are 34 stars labelled as RCB or RCB? in the third edition of the General Catalogue of Variable Stars or its first supplement (Kukarkin et al., 1969). So far as I am aware only 17 of these have been confirmed as RCB stars from spectroscopic evidence. Several should certainly be removed from this class on the basis of their spectra (e.g. $\varrho$ Cas (for instance Payne-Gaposchkin, 1963), Z Cir (Feast, 1965), V 973 Oph, V 1860 Sgr, V 731 Sco (Feast, unpublished)). That the class is not even more heterogeneous is due to earlier weeding operations by Herbig and others.

Waters (1966) has given evidence that, in the case of S Aps, minima normally tend to recur at intervals of about 1290 days. On the other hand Sterne (1935) concluded that $\mathrm{R} \mathrm{CrB}$ itself was an 'ideally irregular variable' on the basis of a statistical analysis of 
the lengths of time between successive minima. It would appear to be a rather important matter for our basic understanding of the RCB phenomenon, to know whether any periodicity is involved or whether minima are spaced completely randomly. More work on this problem would therefore be helpful. It is perhaps worth noting that the type of statistical analysis used by Sterne does not entirely preclude the possibility that minima tend to recur with some basic periodicity, the occurrence or absence of individual minima being governed by some probability law.

Despite the few stars available there is evidence for a concentration of the RCB stars towards the galactic plane and the galactic centre. This suggests membership of an old disc population. Eggen (1965) has argued that the proper motion of $\mathbf{R} \mathbf{C r B}$ shows it to be an old disc object. In addition he places it in his Wolf 630 group, an old disc group.

\section{Abundance, Temperatures}

Only two RCB stars have been studied spectroscopically for quantitative abundance analyses. These are R CrB (Searle, 1961) and RY Sgr (Danziger, 1965)*. These two stars are closely similar to one another. They have the spectra of late $F$ type supergiants but with a large overabundance of carbon and a deficiency of hydrogen. For RY Sgr, Danziger finds carbon more abundant than hydrogen $(\mathrm{C} / \mathrm{H}=25)$ and $\mathrm{C} / \mathrm{Fe}=$ 35 times normal. Lithium seems to be very overabundant in both stars (Danziger, 1965; Keenan and Greenstein, 1963). The $C_{2}$ absorption bands are only weakly present in RY Sgr and R CrB. Several other RCB stars (e.g. S Aps), which are apparently much cooler, show very strong $C_{2}$ bands and are generally classed as $R$ type stars. As was originally pointed out by Bidelman (1953), the RCB stars show only $C_{2}$ bands due to the $\mathrm{C}^{12}$ isotope. Many other carbon-rich stars show significant amounts of $\mathrm{C}^{13}$. Attempts have been made to determine spectroscopic masses for the RCB stars but these appear to be quite uncertain (cf. Danziger, 1965).

A particular constraint in devising a model for the RCB stars is the fact that a large range of temperatures is involved, from late $R$ types up to MV Sgr which has the temperature of a $\mathrm{B}$ type star but is still hydrogen poor, carbon rich.

\section{Absolute Magnitudes}

On the basis of the membership of R CrB in the Wolf 630 group, Eggen (1969) finds $M_{v}=-3^{m}$. 1 . Assuming that the star $12^{\prime \prime}$ from RY Sgr is a physical companion, Andrews et al. (1967) deduce $M_{v} \sim-4$ for the variable. Absolute magnitudes can be derived for the three known RCB stars in the LMC (Feast, 1972). A fourth reported RCB in the LMC (HV 12671) does not belong to this class but is almost certainly a symbiotic star (Feast and Webster, 1974). Two of the LMC RCB stars (W Men and HV 12842) have $C_{2}$ bands of similar strength to the F type RCB stars R CrB and RY Sgr and have $M_{v}$ 's of -5 and -4 . The third one (HV 5637) has very strong $\mathrm{C}_{2}$ bands

* Recently a spectroscopic analysis of XX Cam has been carried out by Orlov and Rodriguez $(1974 a, b)$. 
(like the $\mathrm{R}$ type RCB star S Aps) and also has $M_{v}=-4$. These results indicate that the RCB stars have supergiant luminosities and that there is probably little variation of $M_{v}$ with $\mathrm{C}_{2}$ band strength.

\section{Minima}

The 1948 deep minimum of R CrB was studied spectroscopically by Herbig (1949, see also 1958). Some high dispersion plates obtained during the 1960 deep minimum of R CrB were studied by Payne-Gaposchkin (1963). The 1967-70 deep minimum of RY Sgr was studied spetroscopically and photometrically by Alexander et al. (1972) (=paper III). The spectroscopic changes that take place are very complex and as yet not fully understood.

The following is a brief summary of the behaviour of RY Sgr during the 1967-70 minimum. The initial drop in light was very rapid - 4 mag. in 26 days. During this drop the normal absorption spectrum was rapidly replaced by a rich emission spectrum. The time scale for the fading of the stellar continuum was $\leqslant 5$ days. The emission spectrum when fully developed was very similar to the absorption spectrum at maximum light but now seen in emission. This included the presence of $\mathrm{CN}$ emission bands. There were differences from a simple reversal of the absorption spectrum: Particularly, high excitation lines (e.g. Mg II, C II) which are found in absorption are not clearly seen in emission (though at some phases they may still be strong enough to fill in the absorption lines in the underlying continuum). The presence of a continuum from $\sim 4000 \AA$ to shorter wavelengths, at this phase, has been attributed to the electron attachment spectrum of $\mathrm{CN}$. This rich emission-line spectrum is probably similar to that discussed by Payne-Gaposchkin (1963) in R CrB. She derived a lower temperature and a much lower density for the emission region than for the normal stellar atmosphere and suggested that the emission region should be termed a chromosphere. Evidently at this stage the stellar photospheric radiation is heavily obscured (or decreased for some other reason) and we see the emission from the outer (chromospheric) region which is unobscured.

The chromospheric emission spectrum gradually fades and also changes with time. The most striking feature is the fading of Fe II emission which dominates the initial chromospheric spectrum. The results indicate that in addition to a decrease in flux, the chromosphere shows a decrease in the effects of self absorption in the emission lines and also a lowering of the level of excitation. During the decline there is a recovery phase which probably just represents an increase in the amount of photospheric radiation received. The appearance of the chromospheric spectrum is not related in any simple way to the magnitude of the star. Qualitatively at least it seems that the changes in the chromospheric spectrum proceed in one direction from the time they are first observed. There is no reversal of spectral change during the recovery phase just mentioned. A similar result was obtained by Payne-Gaposchkin (1963) who noted in $R$ $\mathrm{CrB}$ that the flux in the chromospheric emission seemed to depend on the time from initial decline rather than the magnitude. In both $\mathrm{R} \mathrm{CrB}$ and $\mathrm{RY} \mathrm{Sgr}$ the chromospheric emission has a characteristic time scale for decay of $\sim 20$ days. 
In the case of the 1948 minimum of $\mathrm{R} \mathrm{CrB} \mathrm{(Herbig,} \mathrm{1949)} \mathrm{only} \mathrm{the} \mathrm{later} \mathrm{stages} \mathrm{of}$ chromospheric development (after the fading of Fe II emission) were apparently seen. This is perhaps connected with the fact that the initial rate of decline was slower than in 1960 or in the 1967 minimum of RY Sgr. Presumably in 1949 the earlier stages of chromospheric development were obscured by the strong photospheric radiation at that stage.

It begins to look as though the development of the emission spectrum during deep minimum of RCB stars can be fitted to some general pattern. However, much more work is required to confirm this conclusion. The current deep minimum of $\mathrm{V} \mathrm{CrA}$ is being followed spectroscopically and with $U B V$ and infrared photometry (Andrews, Glass, Feast). Amongst other features, strong $\mathrm{C}_{2}$ bands in emission were found during the early development. The changes in strength of $\mathrm{C}_{2}$ in $\mathrm{R} \mathrm{CrB}$ during minimum (Seeds and Ignatuk, 1973) are probably due to the filling in of the photospheric absorption bands by chromospheric emission.

In discussing the chromospheres of the RCB stars it may be necessary to take backwarming by circumstellar dust into account (cf. Buerger and Collins, 1973).

A point of considerable importance for models of RCB stars is the fact that at some phases during minima shell absorption lines ( $\mathrm{Ca}$ II, $\mathrm{Na}$ I) have been detected with large negative radial velocities $\left(200-250 \mathrm{~km} \mathrm{~s}^{-1}\right)$. Such lines were detected during the initial decline of RY Sgr in 1967. These shell lines were again detected during the rising branch at a stage when there was a marked slowing down in the rate of rise and when the photospheric absorption spectrum appeared to be filled in by chromospheric emission. In both cases it seems that there is obscuration of the photosphere which is accompanied by the ejection of gas from the star. Similar shell lines have been found during a minimum of R CrB (Payne-Gaposchkin, 1963).

A persistent feature of at least the later stages of development of $\mathbf{R} \mathbf{C r B}$ and $\mathrm{RY}$ Sgr in minimum is the presence of broad emission lines of $\mathrm{Ca}$ II ( $\mathrm{H}$ and $\mathrm{K}$ ), $\lambda 3888 \AA$ He I and the D lines (Herbig, 1949; Payne-Gaposchkin, 1963; Paper III). The half widths at half intensity were $\sim 170 \mathrm{~km} \mathrm{~s}^{-1}$ in $\mathrm{R} \mathrm{CrB}$ in 1960 . They seem likely to be a characteristic feature of all RCB stars. $\lambda 3888 \AA$ He I emission was detected during a minimum of RS Tel (Feast and Glass, 1973) and this line together with $\mathrm{H}$ and $\mathrm{K}$ have been present for a long period during the current minimum of $\mathrm{V} \mathrm{Cr} \mathrm{A}$. Payne-Gaposchkin (1963) ascribes them to material ejected from the star with high velocity which is excited by collision with a circumstellar cloud. In R CrB and RY Sgr the lines become prominent one or two months after the initial drop in the case of a major decline (70 days in the case of RY Sgr in 1967, 30 days in the case of $R \mathbf{C r B}$ in 1948). In this length of time material travelling with the velocities indicated by the shell absorption lines $\left(200-250 \mathrm{~km} \mathrm{~s}^{-1}\right)$ will have moved about 4 to $8 \mathrm{AU}$ from the star. This agrees roughly with the radius of the infrared emitting shell as estimated by Lee and Feast (1969) (5-10 AU). This latter value is of course rough because the infrared shell is almost certainly non-uniform. Nevertheless the approximate agreement suggests that Payne-Gaposchkin's model of collisional excitation may be quite plausible. 


\section{Absorption and Reddening by Circumstellar Matter}

$U, B, V$, photometry and radial velocity observations (paper III) on the rising branch of RY Sgr and at maximum show that this star is a pulsating variable with a period of 38.6, confirming the visual observations of Jacchia (1933). In addition it was found that there were phases when the spectrum of the variable resembled closely that at maximum light but the star was faint and red. These phases define reddening lines in the $V$ vs $(B-V)$ and $(U-B)$ vs $(B-V)$ diagrams and yield $A_{V} / E_{B-V}=4.3$ and $E_{U-B} /$ $E_{B-V}=1.3$. This is excellent evidence in favour of the Loreta-O'Keefe hypothesis (Loreta, 1935; O'Keefe, 1939) that (at least at these stages in RY Sgr) the minimum is due to obscuration of the star by a cloud of (carbon) particles. The fact that a good $V$ vs $(B-V)$ relation was obtained strongly suggests that the obscuring material was, at the relevant times, fairly uniformly distributed over the observed hemisphere of the star. Evidently it would be of interest to obtain data at other minima of RY Sgr and of other variables to test for variations in the reddening law. Interesting photometric results on $\mathrm{R} \mathrm{CrB}$ itself were obtained by Fernie et al. (1972). However it is difficult to interpret UBV photometry with certainty unless concurrent spectroscopic data are available. It was clear in the case of RY Sgr that quite spurious conclusions on the reddening law would have been drawn if phases at which emission lines were visible or the spectrum was otherwise peculiar, had not been rejected. Indeed at some phases when RY Sgr was faint, both $(B-V)$ and $(U-B)$ were bluer than at maximum due to the effects of emission lines. In view of these considerations the possibility of neutral absorption as suggested for one phase of $\mathrm{R} \mathrm{CrB} \mathrm{(Fernie} \mathrm{et} \mathrm{al.,} \mathrm{1972)} \mathrm{remains} \mathrm{in} \mathrm{need}$ for further study. If neutral absorption were found it would probably require other types of observation (e.g. polarization) to decide whether this was due to intrinsic properties of the particles at that stage or to the existence of a dense obscuring cloud which only partially covered the star.

\section{Pulsation of RY Sgr}

The light, colour and radial velocity pulsations of RY Sgr maintain a fairly constant period of 38.6 days but are of variable amplitude. Trimble (1972) has shown that models of high-luminosity (evolved) helium stars can be constructed to fit the observations reasonably well. The observations of Paper III can be used to deduce a number of interesting points. Integration of the observed velocity curves during 1970 when they were best observed (and when the star was near maximum light) shows that the total displacement during a cycle is large. Because of the irregularity in the velocity curve, the $\gamma$-velocity cannot be determined with great precision. However for a range of possible $\gamma$-velocities the mean displacement is near $3.4 \times 10^{7} \mathrm{~km}$ and the maximum observed displacement is near $5.7 \times 10^{7} \mathrm{~km}$. In Table I these values are compared with radii calculated for different assumed absolute magnitudes of RY Sgr. We adopt $T_{\text {eff }} \sim 6000^{\circ}$. At this temperature $M_{\mathrm{Bol}} \approx M_{v}$ (the infrared excess, assumed due to a shell, is not relevant to the present discussion). Reasonable variations in $T_{\text {efr }}$ will have 
TABLE I

Radii of RY Sgr for various assumed absolute magnitudes

\begin{tabular}{cc}
\multicolumn{2}{c}{$\left(T_{\text {eff }}=6000 \mathrm{~K}\right)$} \\
\hline$M_{V}$ & Radius $R / R_{\odot}$ \\
\hline-6 & 135 \\
-5 & 85 \\
-4 & 54 \\
-3 & 34 \\
-2 & 21 \\
\hline mean displacement $(\Delta R)$ of \\
atmosphere during pulsa- \\
tion (1970): 48 solar radii \\
maximum displacement \\
( $\Delta R_{\max }$ of atmosphere \\
during pulsation (1970): 82 \\
solar radii
\end{tabular}

only a slight effect on the results. The values of $M_{v}$ in the table cover -5 (the absolute magnitude of W Men, the brightest RCB in the LMC), -4 the value proposed for RY Sgr by Andrews et al. (1967) and -3 the value proposed for R CrB by Eggen (1969). The pulsation amplitude $\Delta R / R$ is about $10-20 \%$ for classical Cepheids and about $50 \%$ for type II Cepheids. Table I shows that for RY Sgr $\Delta R / R$ is $>100 \%$ unless the absolute magnitude is in the upper part of the range of the table. The results suggest that absolute magnitudes of -4 or brighter are probably more physically realistic than lower absolute magnitudes. Even at the brighter absolute magnitudes $\Delta R / R$ is still very large (not much less than $100 \%$ for $\Delta R_{\max }$ ). Large amplitudes are characteristic of some of the models of Trimble (as they are of Christie's models for type II Cepheids to which Trimble's helium star pulsation models bear some resemblance). Since these models are based on assumed low masses (1-2 $\mathfrak{M}_{\odot}$ ), this gives support to the idea that $R Y$ Sgr is indeed a low mass star and an evolved member of the old disc population.

The large pulsation amplitude and the irregularity in the pulsational behaviour suggests that the star may be sufficiently unstable to eject matter. Again this would be consistent with Trimble's calculations. She found that her $1 \mathfrak{M}_{\odot}$ model was trying hard to blow itself apart. In these circumstances it is very tempting to suggest that the RCB phenomena (the minima) are triggered by the ejection of matter during a particularly large pulsation. It has however to be remembered that the phenomena near minimum are quite complex, e.g. the high velocity shells.

It would be important to definitely establish whether or not the occurrence of a deep minimum in RY Sgr has any effect on the pulsation. For instance, are the pulsations before and after minimum in phase? Certainly the ejection of a shell on the rise of RY Sgr in 1968 did not affect the phasing (Paper III).

If the minima of RCB stars are triggered by pulsation then all RCB stars should pulsate. Because the light amplitude may be quite small and not very regular, accurate and intensive photometry is necessary to decide this point. There is insufficient data 
to decide in the case of most RCB stars. UW Cen is reported to vary with a period of 42 days (Bateson, 1972). $\mathrm{R} \mathrm{CrB}$ shows small amplitude variations with a period of about 44 days at least at some epochs (Fernie et al., 1972). The cooler star S Aps has a longer period (120 days, Waters, 1966). This longer period may reflect a $P \sqrt{ } \varrho$ relation.

XX Cam seems to have a small amplitude cyclical variation as well as some very small amplitude, very rapid variations (Totochava, 1973). It has been reported (Herbig, 1967) that Miskin observed rapid oscillations in R CrB near minimum. Very high frequency pulsations in R CrB were not detected by Horowitz et al. (1971) in 1969. There are suggestions that the pulsations of RY Sgr may die out from time to time (Mayall, 1972) [and also those of R CrB (Fernie, 1972)]. Also that the pulsation amplitude may be largest during the recovery from minimum for UW Cen (Bateson, 1972).

Even though the light and velocity curves of RY Sgr are not regular it might be thought possible to apply the Baade-Wesselink method to a series of simultaneous spectroscopic and photometric observations such as were obtained near light maximum in 1970 (Paper III). However for a range of $\gamma$-velocities and working as usual with pairs of points of equal $B-V$, one obtains a wide range of radii, a majority of which are negative. Clearly the method fails, at least in its simplest form. Two possible causes for failure in this case are: (1) The radial velocity variations do not reflect the variations in photospheric radius; (2) Phases of equal $(B-V)$ are not phases of equal photospheric temperature. The second possibility might conceivably arise if the variable forms and dissipates particles during a cycle with the formation and dissipation lagging behind the temperature variations.

No spectral type changes round a cycle of RY Sgr have yet been detected with certainty. It would be interesting to place limits on the temperature variations in a cycle, by spectrophotometry of high dispersion spectra. This might establish whether temperature changes can account for the cyclical colour changes or whether particle formation and dissipation through the cycle needs to be postulated.

\section{Infrared}

Infrared excesses in $\mathrm{R} \mathrm{CrB}$ and RY Sgr were discovered at about the same time (Stein et al., 1969; Lee and Feast, 1969). The data indicated a black-body temperature of $\sim 900 \mathrm{~K}$ for the infrared emission. Krishna Swamy (1972) fits models to this data and finds $T \sim 550^{\circ}$. The early data suggested that during minima the loss in light in the visible was compensated by radiation in the infrared, as would be expected from a spherically symmetrical model for a shell of particles. Later work (Forrest et al., 1971) does not support this model. Infrared variations can take place which are uncorrelated with changes in visual magnitude and vice versa. This suggests that a patchy model for the circumstellar dust may be appropriate. Lee (1973) has however cautioned that (unobserved) changes in the long-wavelength infrared emission might affect these conclusions. Feast and Glass (1973) gave evidence that all RCB stars had an infrared excess. [Note that RZ Nor was incorrectly identified at that time. The correct 
star has an infrared excess (Glass, private communication) and the spectrum of a typical RCB star]. MV Sgr, the B type RCB star, also has a cool shell. Glass (1974) has also detected an infrared excess in W Men, the (visually) brightest RCB star in the LMC. The absence of silicate emission in $\mathrm{R} \mathrm{CrB}$ is taken as evidence for the reasonable hypothesis that the dust is made of carbon (e.g. Woolf, 1973). It may be noted here that Snow (1973) found the $4430 \AA$ diffuse interstellar band to be absent in $\mathrm{R} \mathrm{CrB}$ when $\sim 1$ mag below maximum. None of the diffuse interstellar bands were noted as very strong in the Pretoria work on the 1967-70 minimum of RY Sgr though quantitative measures would be desirable to place limits on the band strength.

\section{Polarization}

Serkowski and Kruszewski (1969) observed the polarization of RY Sgr in UBV during a minimum. They found changes in percentage polarization and position angle with time. Coyne and Shawl (1973) observed the polarization of R CrB in UBVGRI during minimum. Polarization measurements of $\mathrm{R} \mathrm{CrB}$ have also been made by Orlov and Rodriguez (1972). Variations are present in percentage polarization, position angle and wavelength dependence. Coyne and Shawl have interpreted these variations in terms of clouds with differing mean particle size dominating the observed polarization at different times. While this appears quite plausible it is difficult (as in the case of the reddening law discussed above) to be certain of the proper interpretation unless details of the spectroscopic behaviour are known at the time of the polarization measurements. For instance if chromospheric emission is present we would anticipate that this would be polarized less than (or at least, differently from) the photospheric radiation. (The photosphere must be more heavily obscured than the chromosphere for the latter to be seen). The relative contributions of chromosphere and photosphere to the total light will in general be a function of wavelength (usually the chromospheric contribution will increase to shorter wavelengths). Thus a wavelength dependence of polarization would result which is unconnected with particle size. It would for instance be particularly interesting to know whether the decrease in polarization at short wavelengths in R CrB just before minimum (in the observations of Coyne and Shawl) could be due to this cause.

In the case of RY Sgr the possible formation and dissipation of particles in the 38.6 day cycle might be investigated by polarimetry.

\section{Models}

The observations of RY Sgr on its rising branch seem to show rather conclusively that for considerable periods at this stage we are observing an essentially normal but dimmed and reddened star. There seems little doubt therefore that at this stage the LoretaO'Keefe hypothesis holds. The dimming is due to particles (presumably of carbon) between the star and the observer. The polarization measurements of $\mathrm{R} \mathrm{CrB}$ and RY Sgr are consistent with this conclusion. It is not yet certain whether the initial 
decline is due to dust obscuration or whether there is an initial decline in light due to some other cause and that this triggers particle formation.

The infrared observations are consistent with the hypothesis of low temperature dust clouds in the systems. The cool $\mathrm{HdC}^{*}$ stars and the hot helium stars are very similar spectroscopically to RCB stars. However they do not undergo deep minima and they do not show infrared excesses (Feast and Glass, 1973). Evidently the occurrence of obscuring dust clouds and of low temperature infrared emission are closely connected. Several alternative models involving dust clouds have been suggested.

Paper III mentioned two possibilities.

(1) The formation of particles in the upper photosphere of the star. The chromosphere lies above the obscuration. Because it is cut off from its source of excitation it gradually decays. This is essentially the model of Payne-Gaposchkin (1963).

(2) The ejection of a cloud from the star towards the observer. The chromospheric spectrum then results from a true eclipse by the dense cloud.

There are a number of points which appear to favour this second alternative. It was noted in Paper III that the development of the chromospheric spectrum with time had considerable similarities to the development of the solar chromospheric spectrum during an eclipse. Since the excitation of the chromosphere remains radiative, the absence of [Fe II] emission is more easily understood (cf. Paper III). The high velocity absorption shells and the broad-lined emission spectrum of $\mathrm{Ca}$ II, $\mathrm{Na}$ I and $\mathrm{He}$ I would presumably be associated with the expanding dust cloud. On this model we might for instance imagine a dense cloud of dust, considerably smaller than the stellar diameter, forming near the star. This would move away from the star and grow in size causing the eclipse. Deep minima would perhaps only occur when the cloud was formed more or less centrally on the observed disc. On this model the time of decline would be related to the rate of growth in size of the cloud and the stellar radius. Since the Magellanic Cloud results suggest little variation of visual absolute magnitude with temperature (as judged from $\mathrm{C}_{2}$ band strength), the cooler stars will have larger diameters. We might therefore expect that the time of decline would be greater for the cooler stars provided the rate of growth of the cloud size is not strongly dependent on the stellar temperature. In fact Payne-Gaposchkin (1963) pointed out that the average time of decline increased with decreasing stellar temperature. It ranges from about 30 days for $\mathrm{R} \mathrm{CrB}$ itself to about 300 days for the coolest RCB stars. On the above proposal this requires the radii of the coolest $\mathrm{RCB}$ stars to be about a factor of 10 larger than that of $\mathrm{R} \mathrm{CrB}$ itself. This would appear reasonable though accurate estimates of the radii of the cooler RCB stars cannot be made since their temperatures are only poorly known.

Forrest et al. (1972) have obtained infrared observations which suggest that clouds are formed which move outwards from the star, cooling and expanding as they do so.

A further point in favour of hypothesis 2 is the possibility that it may be difficult to form particles at photospheric level. Krishna Swamy (1972) estimated that they could

* =hydrogen-deficient carbon stars. 
only be formed at about 7 stellar radii from the surface. Further work on this point would be of value. The formation and acceleration of particles from stars by radiation pressure has been treated by several theorists with conflicting results (e.g. Friedemann and K.-H. Schmidt, 1968; Wickramasinghe, 1972; Gilman, 1972, 1973; Fix and Alexander, 1974).

The fact that drops in light are limited to about $9^{m}$ or less presumably places an upper limit on the size and density of the clouds that can be formed at any one time.

Two other models of the RCB phenomenon have been suggested.

(3) Wing et al. (1972) propose that there are semipermanent blotches in orbit around the star. These give rise to the infrared emission and occasionally pass in front of the star producing a minimum. This model is attractively simple but seems to have a number of difficulties (cf. Feast and Glass, 1973). While there is a good deal of irregularity in RCB light curves they appear in general to have a characteristic asymmetry. There is a rapid decline and a slower rise. Any such asymmetry would hardly be expected on the 'blotch' model. As already noted the $V$ vs $(B-V)$ variations of RY Sgr on the rise suggest a fairly uniform obscuring medium in front of the star at that stage. The observations suggest a gradual thinning of this obscuration. The passage of a very dense, star sized, blotch across the disc will not reproduce the observed colour changes. Finally the association of physical effects (the shell ejection shown by the displaced absorption lines) with the minimum indicates that more is involved than just a geometrical eclipse.

(4) Humphreys and Ney (1974) have put forward essentially the same type of model as Wing et al., except that they associate the particle clouds with the atmosphere of a hypothetical companion star. The basis of this idea is that at $3.5 \mu$ the brightness of $\mathrm{R} \mathrm{CrB}$ has varied relatively smoothly with time between 1968 and 1973 . There was a minimum in 1970 and a maximum in 1971. On this evidence Humphreys and Ney believe that there is a cool long period variable in the $\mathrm{R} \mathrm{CrB}$ system. It will be of great interest to see whether further work confirms a periodicity in the $3.5 \mu$ mag. However the difficulties encountered by model (3), above, also apply in this case. In addition it would appear to require that both stars are carbon rich objects.

At the present time the observations appear to favour hypothesis 1 or 2 rather than 3 or 4 . Possibly hypothesis 2 is the most attractive at the moment but further work will be necessary before a decision can be made with certainty. Presumably matter ejected from the star at minimum replenishes the circumstellar matter which is giving rise to the infrared emission.

\section{Evolutionary State and Related Objects}

The similarity in chemical composition of the RCB stars to the cool, HdC stars and the hot, helium stars obviously suggests a close connection between all these objects. The exact nature of this relationship is not clear (cf. Feast and Glass, 1973). It is perhaps of relevance that Landolt (1973) has found some of the helium stars to be slightly variable in light $(\Delta m \sim 0 m 1)$. The peculiar abundances of these objects have suggested to various workers that the stars have ejected hydrogen rich envelopes 
exposing material which has undergone nuclear processing (cf. the discussion of Warner, 1967). The possibility that these stars have ejected a hydrogen rich shell and the likelihood that most of them belong to an old disc population have suggested a link with the planetary nebulae. The presence of [O II] emission in $\mathrm{R}$ CrB near minimum (Herbig, 1968) suggests surrounding nebulosity in this case. The peculiar variable V 348 Sgr (Herbig, 1958) has similarities in light curve and spectrum to the RCB stars and also has a large infrared excess (Feast and Glass, 1973). It is probably closely related to the RCB stars. Herbig found the object to be involved in nebulosity and suggested a relation to the planetáry nebulae. A connection between RCB stars and the planetary nebulae has been strengthened by the work of Webster and Glass (1974). This places V 348 Sgr in a small group of cool WC stars of the old disc or halo population. The objects are involved in low excitation nebulae and show dust emission in the infrared. Webster and Glass suggest that this group forms an extension of the planetary nebulae with WC nuclei to cooler temperature. Another link between the RCB variables and the planetary nebulae is suggested by the peculiar variable V 605 Aql which is the central star of a planetary nebula (Ford, 1971; van den Bergh, 1971). The variable fluctuated between $10^{m}$ and $12^{m}$ in $1918-1921$ but seems to have been very faint since that time. Evidently it is not a typical RCB variable but Bidelman (1973) has found that it had the spectrum of a hydrogen deficient carbon star when bright and so is probably closely related to the RCB class. It is encouraging to note that the theory of the ejection of planetary nebula shells (e.g. Faulkner, 1970) suggests that a hydrogen poor object will be left behind.

McLaughlin (1935) was apparently the first to suggest that the deep minima characteristic of the transition phase of nova Herculis 1934 (DQ Her) and some other novae, were due to the formation of dust clouds. He also noted a possible connection between the novae and the RCB stars. The spectra of RCB stars have some similarities (e.g. strength of carbon features) to the spectra of slow novae near maximum (e.g. Bidelman, 1954, 1956). The novae also belong to an old disc population. McLaughlin's suggestion has received considerable support recently from the discovery of infrared excesses and polarization in some novae (e.g. Geisel et al., 1970; Zellner and Morrison, 1971 ; Zellner, 1971). Some of the structure of the emission lines in novae can also be understood in terms of a dust model (e.g. Stratton, 1945; McLaughlin, 1949; Hutchings and Fisher, 1973; Malakpur, 1973). The view, discussed earlier, that the ejection of matter from RCB stars may be non-spherical may also be a link with novae since the ejection of matter is known to be non-spherical in that case too.

There are thus strong hints of a connection between the RCB stars and both the planetary nebulae and the novae. However the precise nature of this relationship is not clear.

A considerable amount of theoretical work has been carried out on the evolution of helium rich stars. Some of this work is directed to the problems of the helium main sequence, the white dwarfs and the planetary nebulae rather than specifically to the problem of the RCB stars. Among papers of special relevance to the RCB problem are: Biermann and Kippenhahn (1971), Paczynski (1971), Trimble and Paczynski 
(1973), Wood and Faulkner (1973), Kozlowski et al. (1973), Sackmann et al. (1974). A detailed review of the theoretical work will not be given here. However the following points have emerged from the computations:

(1) It is possible to evolve helium rich, low mass stars into the region of the HR diagram occupied by the RCB stars. In particular stars of about the correct luminosity $\left(M_{v} \sim-5\right)$ can be obtained.

(2) There may still be problems in understanding the carbon abundance (e.g. Trimble and Paczynski, 1973).

(3) Most workers have thought in terms of the ejection of a hydrogen rich envelope. This would take place either in a planetary nebula phase or in some parallel phase (Wood and Faulkner, 1973). However Paczynski (1971) considers the formation of an RCB star without loss of the envelope. He relies on extensive mixing of the hydrogen rich envelope with matter from the interior.

(4) Biermann and Kippenhahn (1971) note that their models of RCB stars have extensive outer convection zones. This suggests that the pulsations of RY Sgr, etc. are, physically, more closely related to the pulsations of Mira variables than to those of the classical Cepheids (despite the fact that the temperature of RY Sgr is similar to that of the Cepheids).

\section{Acknowledgements}

I am grateful to Dr T. Lloyd Evans for reading this paper at the Moscow meeting. I am also indebted to Dr L. Balona for some discussions on the Baade-Wesselink method and to Mrs M. L. Clayton for help in the numerical work.

\section{References}

Alexander, J. B., Andrews, P. J., Catchpole, R. M., Feast, M. W., Lloyd Evans, T., Menzies, J. W., Wisse, P. N. J., and Wisse, M.: 1972, Monthly Notices Roy. Astron. Soc. 158, 305 (Paper III).

Andrews, P. J., Catchpole, R. M., Feast, M. W., Jones, D. H. P., Lloyd Evans, T., and Walker, E. N.: 1967, Info. Bull. Variable Stars, No. 235.

Bateson, F. M.: 1972, Info. Bull. Variable Stars, No. 661.

Bidelman, W. P.: 1953, Astrophys. J. 117, 25.

Bidelman, W. P.: 1954, Trans. IAU 8, 852.

Bidelman, W. P.: 1956, Vistas in Astronomy 2, 1428.

Bidelman, W. P.: 1973, Bull. Am. Astron. Soc. 5, 442.

Biermann, P. and Kippenhahn, R.: 1971, Astron. Astrophys. 14, 32.

Buerger, P. B. and Collins, G. W.: 1973, Bull. Am. Astron. Soc. 5, 348.

Coyne, G. V. and Shawl, S. J.: 1973, Astrophys. J. 186, 961.

Danziger, I. J. : 1965, Monthly Notices Roy. Astron. Soc. 130, 199.

Eggen, O. J.: 1965, Observatory 85, 191.

Eggen, O. J.: 1969, Publ. Astron. Soc. Pacific 81, 553.

Faulkner, D. J.: 1970, Astrophys. J. 162, 513.

Feast, M. W.: 1965, Info. Bull. Variable Stars, No. 87.

Feast, M. W.: 1972, Monthly Notices Roy. Astron. Soc. 158, 11 p.

Feast, M. W. and Glass, I. S.: 1973, Monthly Notices Roy. Astron. Soc. 161, 293.

Feast, M. W. and Webster, B. L. : 1974, Monthly Notices Roy. Astron. Soc. 168, 31 p.

Fernie, J. D.: 1972, Q. J. Roy. Astron. Soc. 13, 81.

Fernie, J. D., Sherwood, V., and Dupuy, D. L.: 1972, Astrophys. J. $172,383$.

Fix, J. D. and Alexander, D. R.: 1974, Astrophys. J. 188, L91.

Ford, H. C. : 1971, Astrophys. J. 170, 547. 
Forrest, W. J., Gillett, F. C., and Stein, W. A. : 1971, Astrophys. J. 170, L29.

Forrest, W. J., Gillett, F. C., and Stein, W. A.: 1972, Astrophys. J. 178, L129.

Friedemann, C. and Schmidt, K-H.: 1968, Astron. Nachr. 290, 233.

Geisel, S. L., Kleinmann, D. E., and Low, F. J. : 1970, Astrophys. J. 161, L101.

Gilman, R. C. : 1972, Astrophys. J. 178, 423.

Gilman, R. C.: 1973, Monthly Notices Roy. Astron. Soc. 161, 3p.

Glass, I. S. : 1974, Monthly Notices Roy. Astron. Soc. 168, 249.

Herbig, G. H.: 1949, Astrophys. J. $110,143$.

Herbig, G. H.: 1957, Etoiles à raies d'èmission, Liège Colloquium,-p. 251.

Herbig, G. H.: 1958, Astrophys. J. 127, 312.

Herbig, G. H.: 1968, Les Transitions interdites dans les spectres des Astres, Liège Colloquium, p. 353.

Herbig, G. H.: 1967, Trans. IAU A13, 530.

Horowitz, P., Papliolios, C., and Carleton, N. P.: 1971, Astrophys. J. 163, L5.

Humphreys, R. M. and Ney, E. P.: 1974, Astrophys. J. 190, 339.

Hutchings, J. B. and Fisher, W. A.: 1973, Publ. Astron. Soc. Pacific 85, 122.

Jacchia, L.: 1933, P. Oss. Ast. U. Bol. $2,173$.

Keenan, P. C. and Greenstein, J. L.: 1963, Perkins Contrib. Series, II, No. 13.

Kozlowski, M., Paczynski, B., and Popova, A. : 1973, Acta Astron. 23, 263.

Krishna Swamy, K. S.: 1972, Publ. Astron. Soc. Pacific 84, 64.

Kukarkin, B. V., Kholopov, P. N., Efremov, Y. N., Kukarkina, N. P., Kurochkin, N. E., Medvedeva, G. I., Perova, N. B., Fedorovich, V. P., and Frolov, M. W.: 1969, General Catalogue of Variable Stars, third edition and first Supplement, Moscow.

Landolt, A. U.: 1973, Publ. Astron. Soc. Pacific 85, 661.

Lee, T. A. and Feast, M. W.: 1969, Astrophys. J. 157, L173.

Lee, T. A.: 1973, Publ. Astron. Soc. Pacific 85, 637.

Loreta, E.: 1934, Astron. Nachr. 254, 151.

Malakpur, I.: 1973, Astron. Astrophys. 28, 393.

Mayall, M. W.: 1972, J. Roy. Astron. Soc. Can. 66, 233.

McLaughlin, D. B.: 1935, Publ. Am. Astron. Soc. 8, 145.

McLaughlin, D. B.: 1949, Publ. Astron. Soc. Pacific 61, 74.

O'Keefe, J. A.: 1939, Astrophys. J. 90, 294.

Orlov, M. Ya. and Rodrìguez, M. H.: 1972, Info. Bull. Variable Stars, No. 742.

Orlov, M. Ya. and Rodrìguez, M. H.: 1974a, Soviet Astron. - AJ 17, 615.

Orlov, M. Ya. and Rodrìguez, M. H.: 1974b, Astron. Astrophys. 31, 203.

Paczynski, B.: 1971, Acta Astron. 21, 1.

Payne-Gaposchkin, C.: 1963, Astrophys. J. 138, 320.

Sackmann, I.-J., Smith, R. L., and Despain, K. H. : 1974, Astrophys. J. 187, 555.

Searle, L.: 1961, Astrophys. J. 133, 531.

Seeds, M. A. and Ignatuk, N.: 1973, Bull. Am. Astron. Soc. 5, 344.

Serkowski, K. and Kruszewski, A.: 1969, Astrophys. J. 155, L15.

Snow, T. P.: 1973, Publ. Astron. Soc. Pacific 85, 590.

Stein, W. A., Gaustad, J. E., Gillett, F. C., and Knacke, R. F. : 1969, Astrophys. J. 155, L3.

Sterne, T. E. : 1935, Harvard Bull., 896.

Stratton, F. J. M.: 1945, Monthly Notices Roy. Astron. Soc. 105, 275.

Totochava, A. G.: 1973, Astron. Circ. (U.S.S.R.), No. 791, p. 7

Trimble, V.: 1972, Monthly Notices Roy. Astron. Soc. 156, 411.

Trimble, V. and Paczynski, B.: 1973, Astron. Astrophys. $22,9$.

van den Bergh, S.: 1971, Publ. Astron. Soc. Pacific 83, 819.

Warner, B.: 1967, Monthly Notices Roy. Astron. Soc. 137, 119.

Waters, B. H. J.: 1966, R.A.S. New Zealand Circ., 119.

Webster, B. L. and Glass, I. S. : 1974, Monthly Notices Roy. Astron. Soc. 166, 491.

Wickramasinghe, N. C. : 1972, Monthly Notices Roy. Astron. Soc. 159, 269.

Wing, R. F., Baumert, J. H., Strom, S. E., and Strom, K. M.: 1972, Publ. Astron. Soc. Pacific 84, 646.

Wood, P. R. and Faulkner, D. J.: 1973, Astrophys. J. 181, 147.

Woolf, N. J. : 1973, Astrophys. J. 185, 229.

Zellner, B.: 1971, Astron. J. 76, 651.

Zellner, B. and Morrison, N. D.: 1971, Astron. J. 76, 645. 